\title{
A biomechanical analysis of the Ilizarov external fixator
}

\author{
A. S. Mitousoudis ${ }^{1}$, E. A. Magnissalis ${ }^{2}$, S. K. Kourkoulis ${ }^{1, a}$ \\ ${ }^{1}$ School of Applied Mathematical and Physical Sciences, Dept. of Mechanics, National Technical \\ University of Athens, 5 Heroes of Polytechnion Avenue, 15773 Zografou, Athens, Greece \\ ${ }^{2}$ Laboratory for the Research of the Musculoskeletal System (LRMS), University of Athens, KAT \\ Hospital, Kifissia, Athens, Greece
}

\begin{abstract}
The objective of the present study is the construction of a reliable numerical model of the standard Ilizarov fixator that will permit a detailed parametric investigation of various factors influencing the effectiveness of the technique. Mechanical tests of a standard Ilizarov frame were performed under axial and torsional loads. To eliminate the pretension loss due to wire slippage metal nuts with M1.8 thread were manufactured. A numerical analysis followed based on the Finite Element Method. The model constructed simulated accurately all the geometrical details of the experimental model, the load application mode (axial and torsional) and the mechanical characteristics of the materials used to assemble the frame. Comparison of the predictions of the model with the respective experimental data yields excellent agreement for the case of tension and rather satisfactory one for torsion.
\end{abstract}

\section{General}

The Ilizarov external fixator is a wire circular frame device invented by Gavriil Ilizarov in Kurgan, Siberia in 1952 [1]. The method relies on the basic bio-mechanical principle of axial compressive loads and micro movement stimulating biological bone bridging of the fracture gap. This mechanism depends on wire tension. The Ilizarov method was not brought to the west until 1981. The principles of Ilizarov external fixator are very different from those of anatomical reduction and rigid internal fixation, enabling success where other methods may fail. Its fundamental difference from similar ones is the type of pins used for bone fixation, pretensioned from 500 to $1300 \mathrm{~N}$ before being affixed to the rings that are connected and fixed by threaded rods (Figure 1). The mechanical characteristics of external fixators may influence the biologic environment at the fracture site and ultimately dictate (or determine) the outcome of a surgical procedure. Thus, knowledge of the mechanical properties of the Ilizarov fixator is essential for the surgeons using it in clinical practice.

It is obvious that the role of the inter-fragmental movements is crucial for the complex process of healing any fracture. Nowadays the most beneficial environment for healing a fracture is not precisely described. However it is reasonable to accept that bending and large shear forces as well as large axial movements constitute negative parameters [2-5]. On the contrary small shear and small axial movements are considered beneficial [6,7]. In this direction and in order to promote very small movements of the bone fragments, the Ilizarov frame requires adequate settings. In the literature it is mentioned that bolt-tightening torques in the 10-20 Nm range and wire pre-tensions of the order of $0.98-1.27 \mathrm{kN}$ are considered optimum for stable fixation [8-10].

a e-mail : stakkour@central.ntua.gr

This is an Open Access article distributed under the terms of the Creative Commons Attribution-Noncommercial License 3.0, which permits unrestricted use, distribution, and reproduction in any noncommercial medium, provided the original work is properly cited. 
Among the most serious limitations of Ilizarov technique is the loss of the initial pre-tension of the wires. Unfortunately the exact cause of this phenomenon is still under discussion: Some researchers consider that pre-tension loss is due to slippage $[8,10,11]$, while others support the opinion that material yields [12]. Clearly a combination of material yield and slippage of the wires [13-15] appears to explain better the pre-tension loss.

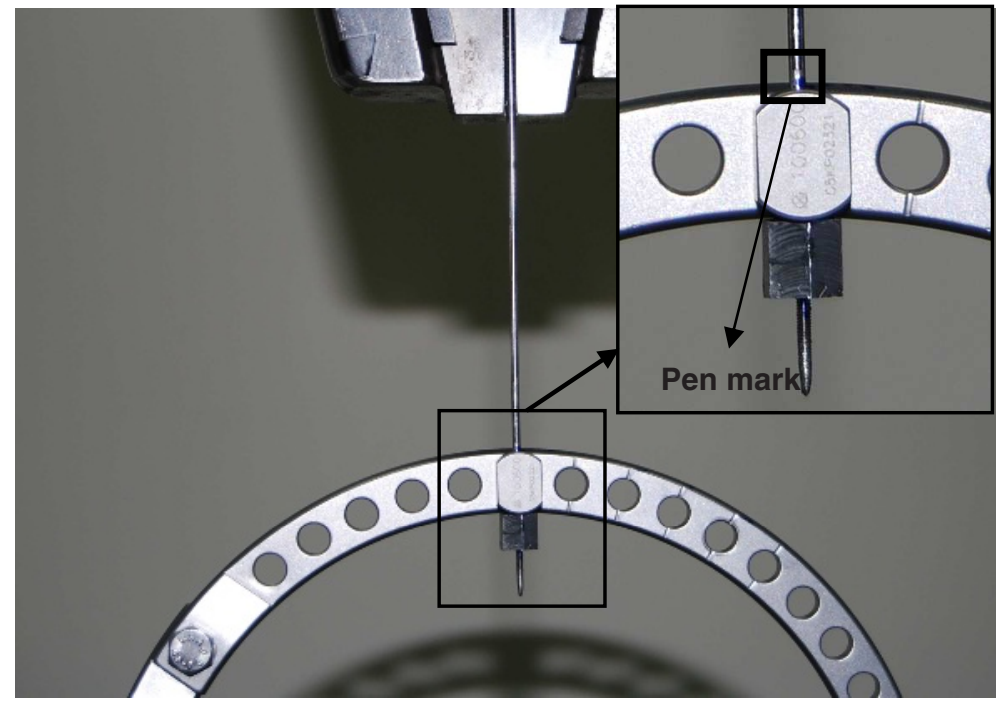

Fig. 1. The configuration prepared to investigate the efficiency of the custom made nut, used to eliminate the loss of pretension due to wire slippage

The objective of the present study is the construction of a reliable numerical model of the standard Ilizarov fixator that will permit the extensive parametric investigation of various factors influencing the effectiveness of the technique. The model will be calibrated and validated using experimental data for different loading conditions. It is mentioned finally that the pretension loss due to wire slippage was completely eliminated by attaching custom made metal nuts to each wire (Figure 1).

\section{Experimental procedure}

To eliminate the pretension loss due to wire slippage in the present series of tests, metal nuts with M1.8 thread were manufactured. Specially designed wires were used with mechanical stoppers (olive-oil drop shaped) at the one side while at the opposite one the threaded nut was used to prevent slippage of the wire through the cannulated bolt. A wire was attached to a metal ring using a cannulated bolt and a threaded nut (Figure 1). A tightening torque of $15 \mathrm{Nm}$ was applied to the nut. The ring was mounted to the base of the loading frame (MTS miniBionix 858) and the wire was attached to the actuator. The wire was pulled away from the metallic ring until failure. The wire slippage was checked by monitoring a pen mark drawn on the wire (Figure 1). The experiment was repeated three times by replacing the wire and the cannulated bolt with a new one. No slippage of the wire was observed during the tests.

Using this procedure, a standard Ilizarov frame was assembled consisting of two rings (diameter $150 \mathrm{~mm}$, Smith \& Nephew part number 101305) and four K-wires with a diameter of $1.8 \mathrm{~mm}$ (Smith $\&$ Nephew part number 102107). The wires in each ring were placed normally to each other (Figure 2). At the one side of the wire, the above described stopper was used to prevent slippage while at the opposite side the threaded nut was attached after the pretension was applied (Figure 2). The wire was attached to the ring using a cannulated bolt (Smith \& Nephew part number 100600). The two rings were connected with four stainless steel threaded rods of length $400 \mathrm{~mm}$ and outside screw thread 
diameter $6 \mathrm{~mm}$ (Smith \& Nephew part number 102314). The bone was simulated by two polyethylene bars, $3 \mathrm{~cm}$ diameter, with a $2 \mathrm{~cm}$ interfragmentary gap between them. A pretension of $110 \mathrm{Kg}$ (1080 Nt) was applied to each wire using an Ilizarov tensioner (Smith \& Nephew part number 103101). A tightening torque of $15 \mathrm{Nm}$ was applied to every nut.

The above described configuration was subjected to axial (along the longitudinal axis of the "bone") and torsional (around the axis of the "bone") loads applied in quasi-static manner. During the tests, displacement / angle and force / torque were recorded continuously.

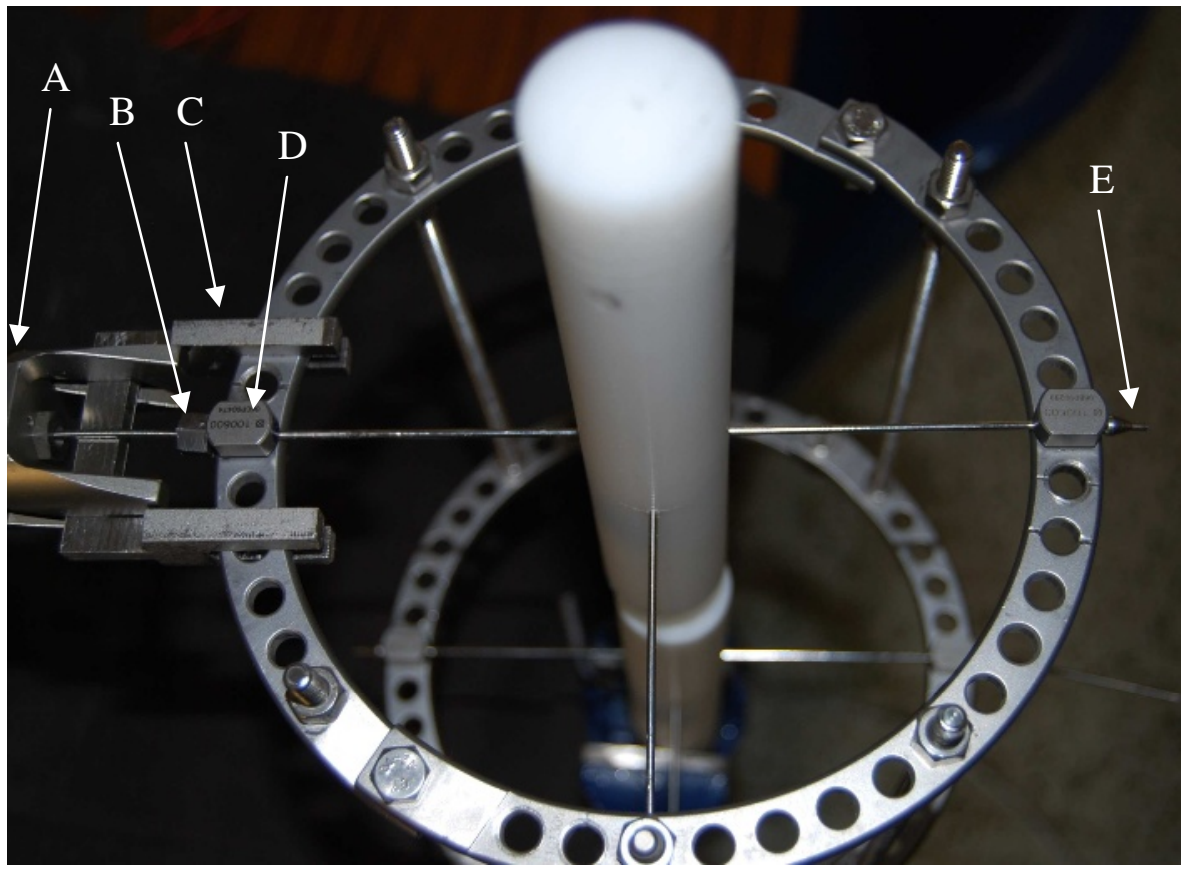

Fig. 2. A: Ilizarov tensioner, B: threaded nut, C: custom made adapter, D: cannulated bolt, E: "olive" type stopper of the wire.

\section{Numerical Analysis}

A numerical analysis followed based on the Finite Element Method in an effort to study in a parametric manner the factors influencing the behaviour and effectiveness of the Ilizarov technique. The numerical model constructed simulated accurately all the geometrical details of the experimental model, the load application mode (axial and torsional) and the mechanical characteristics of the materials used to assemble the frame (stainless steel and acetal).

The ring was modelled as a cylinder with inner and outer diameter $150 \mathrm{~mm}$ and $178 \mathrm{~mm}$ respectively and depth $4.8 \mathrm{~mm}$. Also 44 holes were created to each ring with $6 \mathrm{~mm}$ radius. The threated rod was modelled as a cylinder that did not include the thread with $6 \mathrm{~mm}$ diameter. Both rings and rods were meshed using 8-node prismatic solid elements (solid185) and were modelled as stainless steel with a Young's modulus of $200 \mathrm{GPa}$ and Poisson's ratio of 0.30 . The rings and the rods were rigidly attached. The polyethylene bar was modelled as a cylinder with $30 \mathrm{~mm}$ diameter. Cylindrical holes with $1.8 \mathrm{~mm}$ diameter were created to the polyethylene bar in order for the wires to pass through and to model the bone-wire interface. The wire was modelled as a cylinder with $1.8 \mathrm{~mm}$ diameter. The part of the wire which passes through the bone was meshed with 8-node prismatic solid elements (solid185) and the rest was meshed with 3D quadratic beam elements (beam188).

The material properties for the wire and the polyethylene bar were obtained from experimental data, carried out in the Laboratory of Testing and Materials of the National Technical University of Athens using specimens made from the specific batch. In Figure 3 one can see the experimental 
stress-strain curves used to model the mechanical behaviour of the wire and the polyethylene bar. A multilinear isotropic hardening model was used to import the curve of Figure 3. Poisson's ratio was set equal to 0.3 for the wire and equal to 0.35 for the polyethylene bar.

To model the wire-bone interface, contact elements were used so that the wires were free to slide within the bone, along the wire axis. The area of the holes of the polyethylene bar was meshed with 3D 4-node surface-to-surface contact elements (conta173) and the external area of the solid185 elements of the wire was meshed with 3D target segment elements (targe170). The wire was rigidly attached to the ring. The final model contained 56347 solid 185 elements and 152 beam 188 elements. All nodes on the lower surface of the distal bone were constrained concerning all their degrees of freedom. Finally an axial displacement was induced to all the nodes on the upper surface of the proximal "bone".
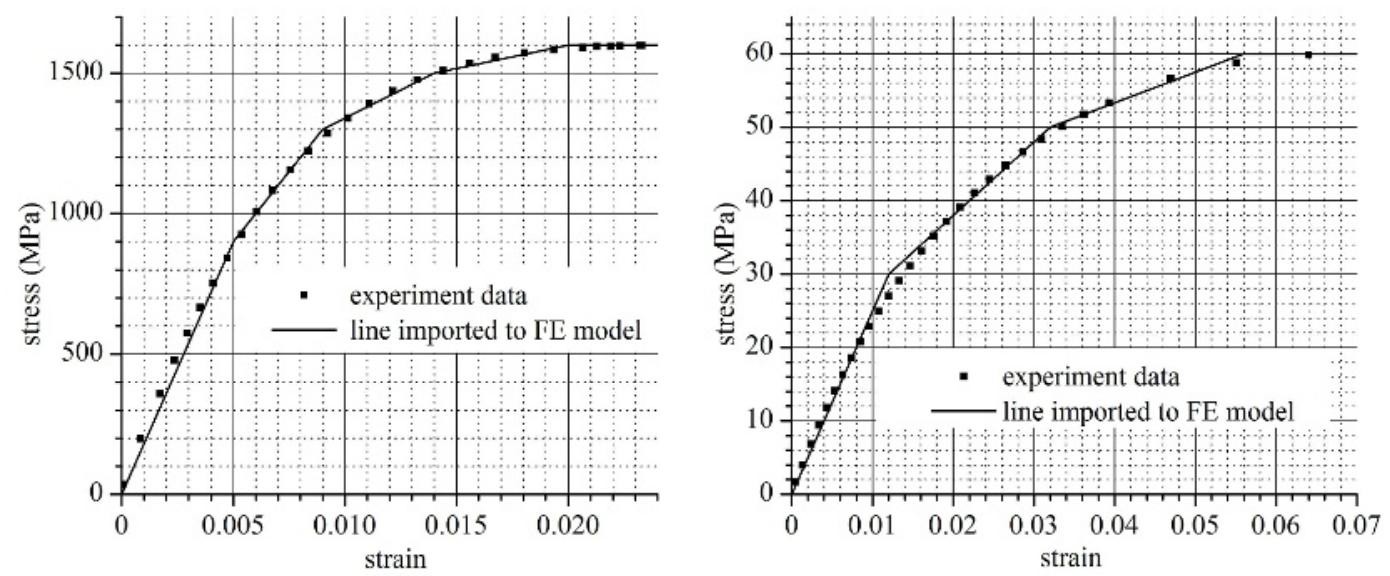

Fig. 3. The experimental stress-strain curves of the wire (on the left) and the polyethylene bar (on the right) and the material properties imported to the FE model.

In order for the configuration of the complete fixator to be accurately defined, for the needs of the numerical analysis, a master input file was created. The location and the orientation of any number of components of the assembly were defined within this file. In addition the instructions required for the interconnection of the various structural members of the fixator were included in this file. Finally data concerning the mechanical properties of the specific materials used, the element types, and the cross-sectional data were also provided to the master file together with information for the loads and the constraints. Any given fixator configuration could be modelled by simply changing the data concerning the location and orientation of the constitutive components making up the specific configuration. In Figure 4 the actual configuration of the fixator used for the mechanical tests is exhibited in juxtaposition to the numerically designed model.

The FE models were solved in multiple load-steps. In the first step the initial pretension was induced to the wires. To do so, a pretension section was created in the middle of each wire and the initial pretension was applied. After the pretension was induced the FE models were loaded to simulate the mechanical testing.

Characteristic results of the numerical analysis are shown in Figure 5(a,b). In Figure 5a the distribution of the von-Mises equivalent stress is plotted for the whole fixator in case it is subjected to axial loading. As it is expected the regions most suspectible to failure are located around the points where the wires are coming in contact with the "bone". Both the wire and the "bone" are under extremely intense stress fields. The components of the fields approach the critical values of the material constants taking into account that the "beneficial" (at least for the relief of materials) influence of the parasitic slippage was totally suppressed in the present study. This point should be studied further since the bearing stresses developed in case of real bones could cause local fracture 
of the tissue. The stress field components in the remaining portions of the fixator are well below the critical values.
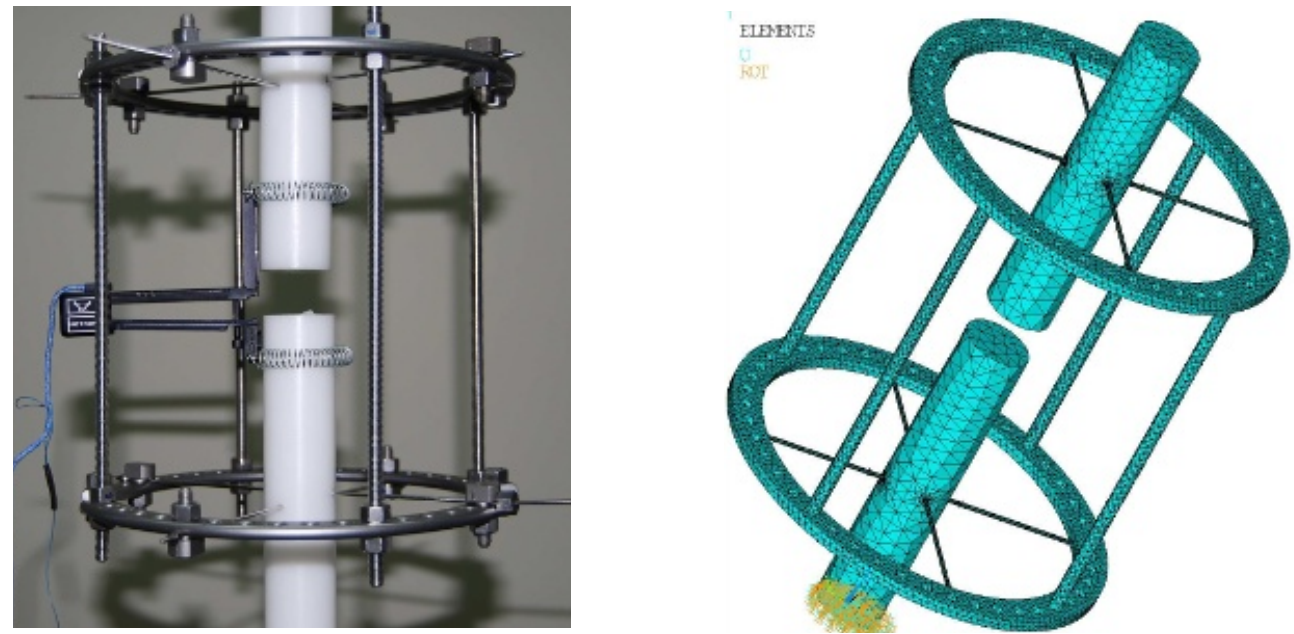

Fig. 4. The Ilizarov fixator during axial load (on the left) and the finite element model of the Ilizarov frame (on the right).

In Figure 5b again the von Mises equivalent stress is shown in case the fixator is subjected to torsion around the axis of the "bone". The conclusions drawn are of similar nature, indicating again that attention should be paid to the region where the wires come in contact with the bone.

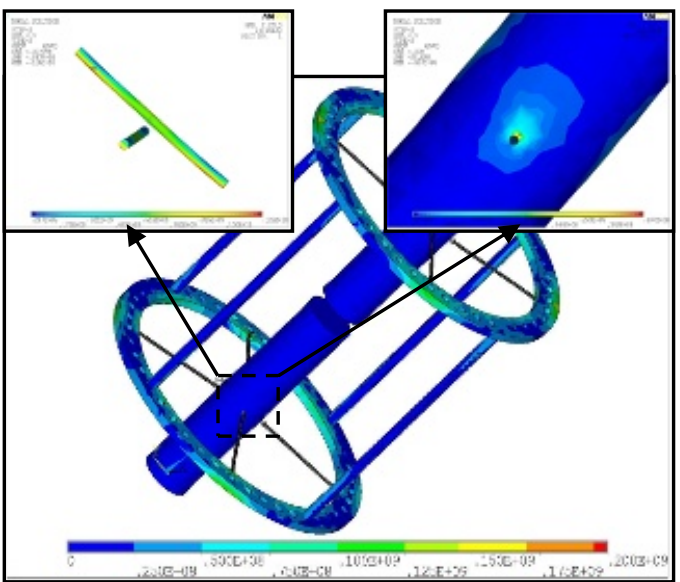

(a)

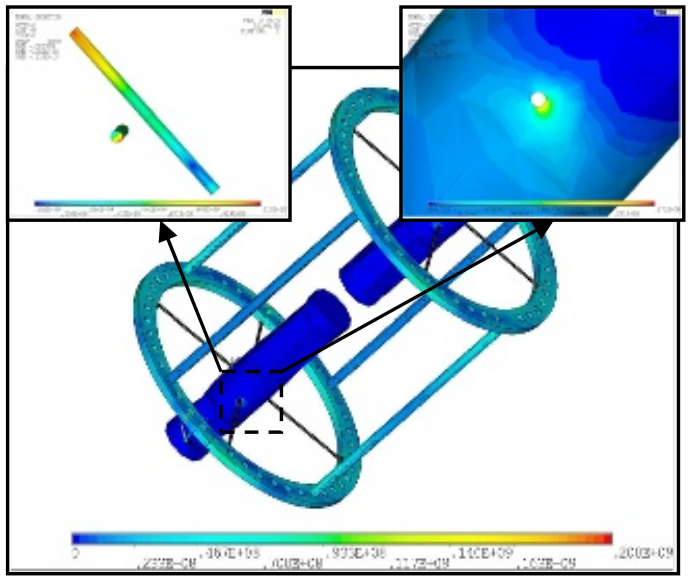

(b)

Fig. 5: The von Mises equivalent stress under axial (a) and torsional (b) loads for the whole Ilizarov frame.

\section{Results and conclusions}

A series of experiments were carried out in order to study the mechanical behaviour of the Ilizarov fixator, in case the pre-tension loss of the wires is completely eliminated. The elimination was achieved using specially designed stoppers and bolts. In addition a numerical model was constructed simulating the mechanical behaviour of the fixator under different elementary load schemes (tension- 
torsion). Comparing the predictions of the model with the respective experimental data (Figure 6) yields an excellent agreement in case of tension and a rather satisfactory one in case of torsion.
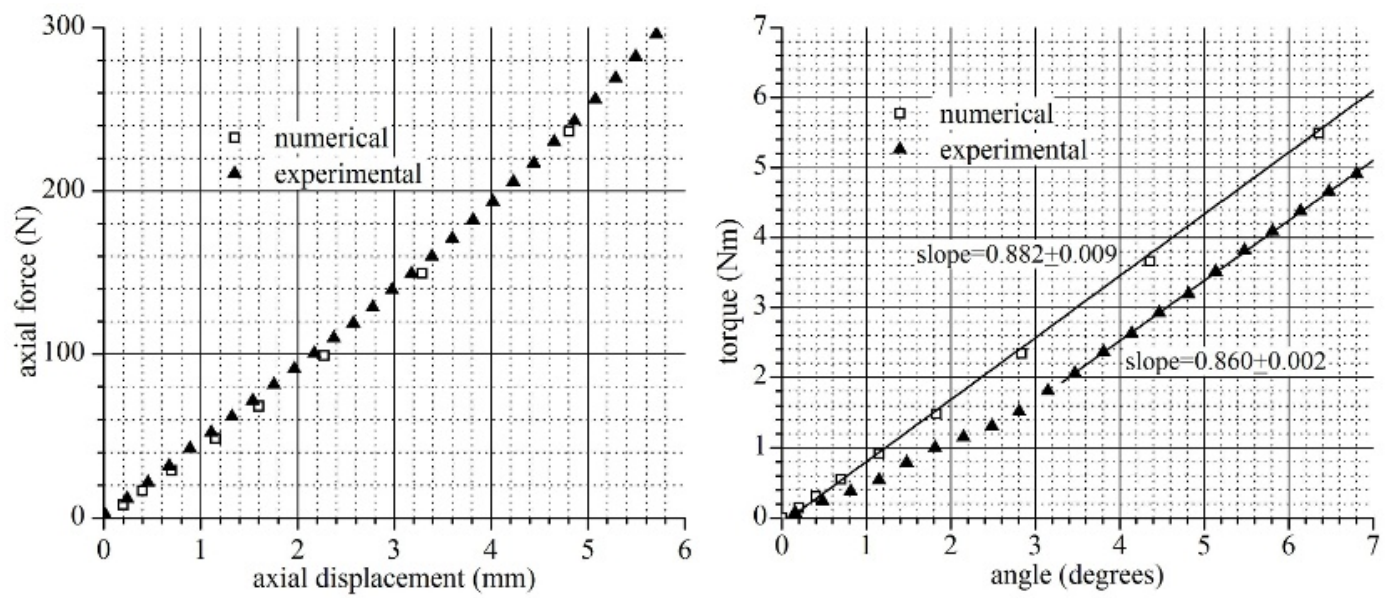

Fig. 6: Experimental and numerical results for axial (on the left) and torsional (on the right) loads of the Ilizarov fixator.

It was concluded that reconsideration of the design of the "bone"-wire interface is required since the stress field developed at this region approaches the nominal values of the allowable mechanical constants. Possible solution could be given by using wires of varying cross area which could be increased in the immediate vicinity of the bone, increasing in turn the contact area.

The above conclusion is further supported by Figure 7(a), where the variation of the von Mises equivalent stress is plotted along the top wire of the assembly in case the fixture is subjected to axial loading. The three curves correspond to the upper, the central and the lower fibers of the wire. It is

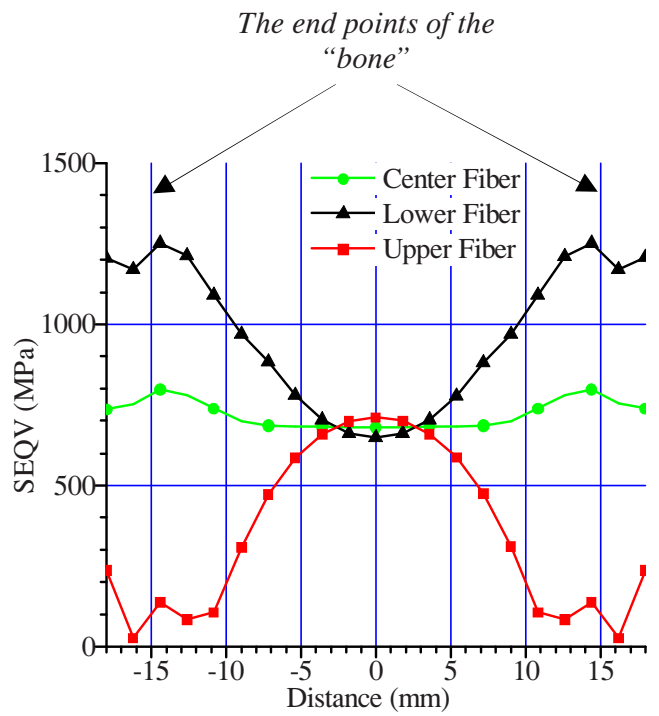

(a)

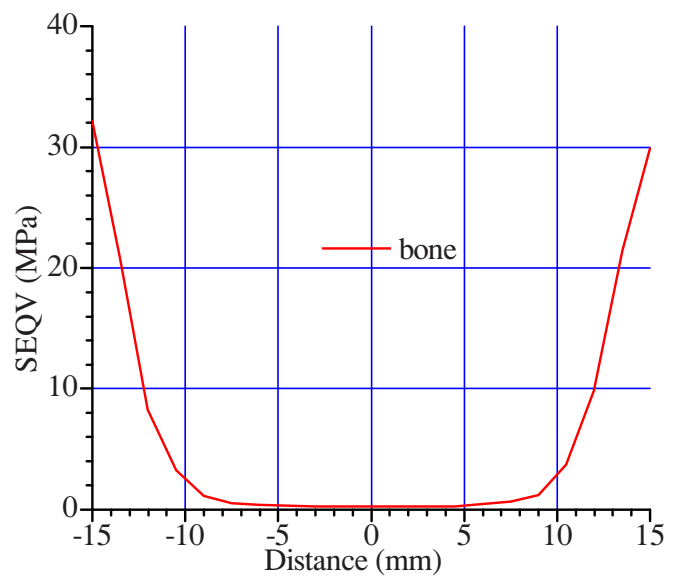

(b)

Fig. 7: The variation of the von Mises equivalent stress along the wire (a) and along the bony surface in contact with the wire (b) in case of axial loading 
clearly seen that the wires are under an extremely intense stress field the maximum values of which exceed $1.2 \mathrm{GPa}$ (!) for the lower portion of the wire. This should be expected since at this region of the wire the pre-existing tensile field (due to the pretension imposed to the wires) is intensified by the presence of the tensile field due to the exerted bending. The central fiber (corresponding more or less to the neutral bending axis) is under constant tensile stress, due only to the pretension. It is also observed that the stress variation along the wire exhibit strong fluctuations and the maximum values appear at the end-points of the "bone" (indicated by arrows in Figure 7a). As one moves towards the center of the wire the stresses decrease since the contact between wire and "bone" is lost. This is more clearly observed in Figure $7 \mathrm{~b}$ where the equivalent stress is plotted along the line of the bone initially in touch with the wire. The stress reaches maximum values at the outermost points of the "bone" and then they are almost zeroed since the wire is not any more in contact with the bone.

Similar conclusions can be drawn from Figure 8a, where the same as above quantities are plotted for the same critical lines, assuming that the assembly is subjected to torsion. The only difference is that instead of plotting along the "upper" and "lower" fibers of the wire the quantities are plotted along the "right" and the "left" ones, as on observes the vertically positioned fixture along the top wire. Clearly the graphs along these lines are "anti-symmetric" (instead of symmetric). The stress field is again very intense and the maximum values exceed 1.1 GPa (again at the end points of the "bony" surface. The stresses along the line of the "bone" initially in contact with the wire are plotted in Figure 8b, and it is again conclude that they are rapidly attenuating as one moves towards the center of the "bone" section.

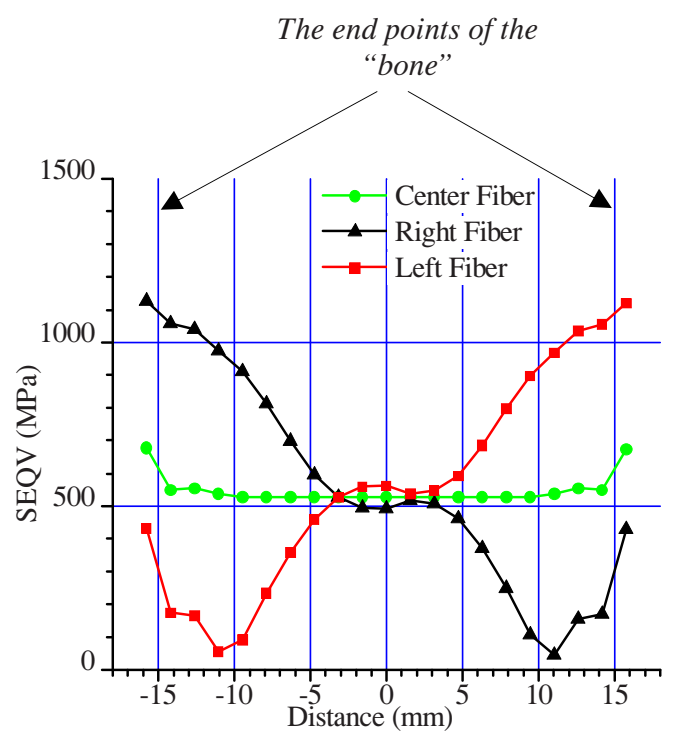

(a)

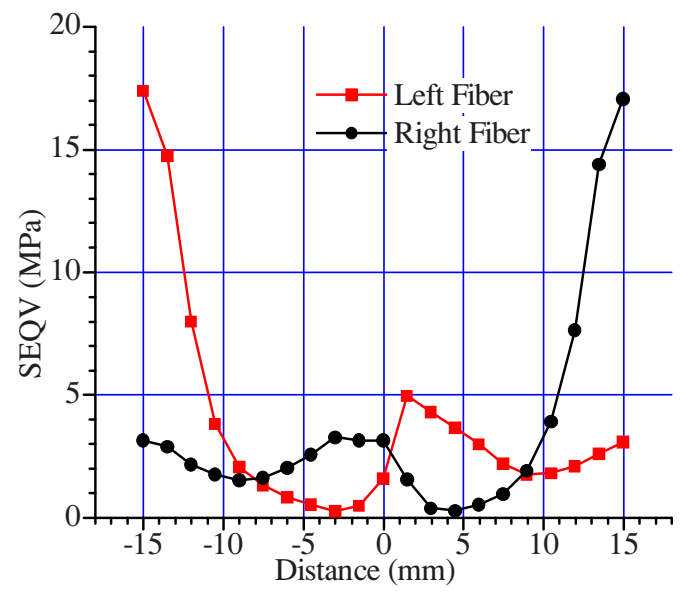

(b)

Fig. 8: The variation of the von Mises equivalent stress along the wire (a) and along the bony surface in contact with the wire (b) in case of torsion

Finally it is concluded that the whole fixator could be redesigned taking into account that the stress field all over the structure (except the wire-bone interfaces) is very weak for the loads expected under normal conditions. Such an approach could reduce the weight of the fixator as well as its cost. In any case it should be always kept in mind that it is the clinical reality and the experience of the surgeons that governs the successful application of the method, since a long series of parameters and problems confronted during the operation can not be predicted and taken into account by the laboratory or numerical simulations. 


\section{References}

1. B. Gasser, B. Boman, D. Wyder, E. Schneider, J Biomech Eng 112, 15-21 (1990)

2. M.H. Noordeen, C.B. Lavy, N.S. Shergill, J.D. Tuite, A.M. Jackson, J Bone Joint Surg Br 77, 645-648 (1995)

3. T. Yamaji, K. Ando, S. Wolf, P. Augat, L. Claes, J Orthop Sci 6, 571-575 (2001)

4. P. Augat, J. Burger, S. Schorlemmer, T. Henke, M. Peraus, L. Claes, J Orthop Res 21, 10111017 (2003)

5. H. Schell, D.R. Epari, J.P. Kassi, H. Bragulla, H.J. Bail, G.N. Duda, J Orthop Res 23, 1022$1028(2005)$

6. S. Wolf, A. Janousek, J. Pfeil, W. Veith, F. Haas, G. Duda, L. Claes, Clin Biomech (Bristol, Avon) 13, 359-364 (1998)

7. N.E. Bishop, M. van Rhijn, I. Tami, R. Corveleijn, E. Schneider, K. Ito, Clin Orthop Relat Res 443, 307-314 (2006)

8. J. Aronson, J.H. Harp, Jr., Clin Orthop Relat Res 23-29 (1992)

9. M.M. Mullins, A.W. Davidson, D. Goodier, M. Barry, Injury 34, 155-157 (2003)

10. A.J. Renard, B.G. Schutte, N. Verdonschot, A. van Kampen, Clin Biomech (Bristol, Avon) 20, 1126-1130 (2005)

11. R. Aquarius, A. Van Kampen, N. Verdonschot, Acta Orthop 78, 654-660 (2007)

12. P.J. Hillard, A.J. Harrison, R.M. Atkins, Proc Inst Mech Eng H 212, 37-47 (1998)

13. C. Delprete, M.M. Gola, J Biomech Eng 115, 37-42 (1993)

14. M.A. Watson, K.J. Matthias, N. Maffulli, D.W. Hukins, Proc Inst Mech Eng H 217, 367-374 (2003)

15. N.A. Osei, B.M. Bradley, P. Culpan, J.B. Mitchell, M. Barry, K.E. Tanner, Injury 37, 941-945 (2006) 